\title{
Emergência e crescimento de plântulas de bacabi (Oenocarpus mapora Karsten) e bacaba (Oenocarpus distichus Mart.) e estimativas de parâmetros genéticos
}

\author{
Ricardo Alexandre Moraes da SILVA¹, Milton Guilherme da Costa MOTA², João Tomé de FARIAS NETO³ \\ RESUMO \\ Foi estudada a variabilidade genética em progênies de bacabi (Oenocarpus mapora Karsten) e bacaba (Oenocarpus distichus Mart.) \\ para caracteres de emergência e crescimento inicial de mudas. Foram coletados frutos maduros de 47 matrizes em quintais \\ de produtores rurais e no Banco de Germoplasma da Embrapa Amazônia Oriental, envolvendo análise de 47 progênies de \\ polinização aberta delineadas em blocos ao acaso, com quatro repetições e parcelas de 24 sementes. As variáveis avaliadas foram: \\ a percentagem de emergência (PE), tempo médio de emergência (TME), taxa de crescimento absoluto da altura de plântulas \\ (TCAA) e taxa de crescimento absoluto do diâmetro de plântulas (TCAD). As progênies apresentaram diferenças significativas \\ $(\mathrm{p} \leq 0,01)$ para todos os caracteres da análise das duas espécies, com boa precisão experimental. Para a herdabilidade no sentido \\ amplo foi verificado que no conjunto total de progênies e no conjunto de progênies de bacabi todos os caracteres estudados \\ apresentaram estimativas de herdabilidade acima de 50\%. As exceçôes foram para TME e TCAD, no conjunto de progênies \\ de bacaba, com estimativas de herdabilidade abaixo de $50 \%$. Os coeficientes " $b$ " apresentaram a mesma tendência observada \\ na herdabilidade, e suas magnitudes expressam também a variabilidade genética. Os ganhos de seleção estimados a partir das \\ porcentagens de seleção pré-determinados mostraram valores altos para todos os caracteres, favorecidos pela pressão de seleção \\ com uma intensidade de $10 \%$ para o total de progênies e apenas bacabi e $20 \%$ para as progênies somente de bacaba.
}

PALAVRAS-CHAVE: Oenocarpus, Emergência, Herdabilidade, Parâmetro genéticos.

\section{Emergence and growth of seedling of bacabi (Oenocarpus mapora Karsten) and bacaba (Oenocarpus distichus Mart.) and estimates of genetics parameters}

\begin{abstract}
It was studied genetic variability in the progenies of bacabi ( Oenocarpus mapora Karsten) and bacaba (Oenocarpus distichus Mart.) for the characters of emergency and initial growth of the changes. One collected mature fruits of 47 matrices in yards of rurais producers yards and from the Germoplasm Bank of the Embrapa Amazônia Oriental in Belém, involving the study of 47 progenies open polinization delineated in blocks experimental design with four replications and parcels of 24 seeds. The variances analyzed were: the emergency percentage (PE), the average time of emergency (TME), the rate of absolute growth of the height of seedling (TCAA) and rate of absolute growth of the diameter of seedling (TCAD). The progenies showed significant statistic differences $(\mathrm{p} \leq 0,01)$ for all the analyzed characters for the two species, with excellent experimental precision. In general, for herdability, it was verified that in the total set of progenies and the set of progenies of bacabi all the studied characters had presented estimates of herdability above of $50 \%$. The exceptions had been for TME and TCAD, in the set of progenies of bacaba, with estimates of herdability below of 50\%. The "b" coefficients showed the same trend observed in herdability, and their magnitudes also express the genetic variability. The gains of selection available take off the percentages of the pre-determined selection showed values highest for all the characters, were auspicious for the pression of the selection with intensity of the $10 \%$ for the total of progenies and alone bacabi and $20 \%$ to progenies of the bacaba.
\end{abstract}

KEY WORDS: Oenocarpus, Emergence, Herdability, Genetic parameters.

\footnotetext{
1 Universidade Federal Rural da Amazônia - UFRA, E-mail: agro_rico@hotmail.com

2 Universidade Federal Rural da Amazônia - UFRA, Av. Tancredo Neves, 2501, Terra Firme, CP 917, CEP 66077-530, Belém, PA, Brasil, E-mail: mota@amazon.com.br

${ }^{3}$ Embrapa Amazônia Oriental, Trav. Dr. Enéas Pinheiro, Marco, CP 48, CEP 66095-100, Belém, PA, Brasil, E-mail: tome@cpatu.embrapa.br
} 


\section{INTRODUÇÃO}

As espécies do gênero Oenocarpus são originárias e nativas da América Tropical e a maioria das espécies ocorre no Norte da América do Sul, indo do nível do mar até $1000 \mathrm{~m}$ de altitude (Lleras et al., 1983).

A importância socioeconômica destas palmeiras é inegável, e na Amazônia são utilizadas pelo homem para diferentes finalidades tais como: habitação, alimentação, ornamentação, medicina popular e confecção de cestaria (Oliveira et al., 1991; Almeida \& Silva, 1992).

Porém, o principal interesse nas espécies Oenocarpus mapora Karsten (bacabi) e Oenocarpus distichus Mart. (bacaba) reside no aproveitamento de seus frutos para produção de óleo e outros produtos derivados, de excelente qualidade nutricional. As propriedades organolépticas do óleo são semelhantes às do óleo de oliva, podendo ser um substituto deste azeite, além de propiciar matéria prima de boa qualidade para a indústria de alimentos pois contém proteínas de excelente valor biológico (40\% a mais que a soja, Balick, 1986).

A emergência de sementes de palmeiras é geralmente caracterizada por dificuldades que variam desde as características morfológicas da semente até as peculiaridades fisiológicas do processo germinativo. As sementes são extremamente sensíveis ao dessecamento, o que ocasiona perda de sua viabilidade reduzindo de forma significativa a sua germinação (Pinheiro, 1986).

Embora as sementes de palmeiras apresentem sinais de dormência, há indícios de que o crescimento embrionário é contínuo e lento assim como o crescimento das plântulas, esta característica parece está relacionada à sua capacidade de armazenar as reservas orgânicas e, posteriormente mobiliza-las para o seu crescimento (Cunha \& Jardim, 1995).

Frutos das espécies do complexo Oenocarpus e Jessenia submetidos à imersão em água morna por meia hora, seguida de remoção do pericarpo apresentam a emergência e o desenvolvimento inicial favorecidos, elevando o percentual total de sementes germinadas em relação aos frutos sem tratamento em um mesmo período (Balick, 1992).

Posiçôes distintas de semeadura não exercem influência sobre a emergência de plântulas de $O$. mapora, alcançando valores acima de $92 \%$; quanto ao vigor, as sementes germinam em menor tempo quando postas com o poro germinativo voltado para a superfície e com rafe na posição horizontal voltada para cima (Nascimento et al., 2001).

Diante da inexistência de informações sobre a emergência e o crescimento inicial de plântulas de bacabi e bacaba, este trabalho teve por objetivo estudar os aspectos fisiológicos relacionados a este processo em progênies de polinização aberta de bacabi e bacaba e estimativas de parâmetros genéticos de modo a subsidiar estudos de variabilidade genética das espécies.

\section{MATERIAL E MÉTODOS}

A coleta de frutos das matrizes de $O$. mapora (bacabi) $e O$. distichus (bacaba) foram realizados em quintais de produtores rurais e no banco de germoplasma da Embrapa Amazônia Oriental. As posições geográficas das cinco localidades correspondem aos municípios sedes de Belém, Colares, Moju, Portel e Santo Antônio do Tauá (situados no Estado do Pará), como mostra a Figura 1.

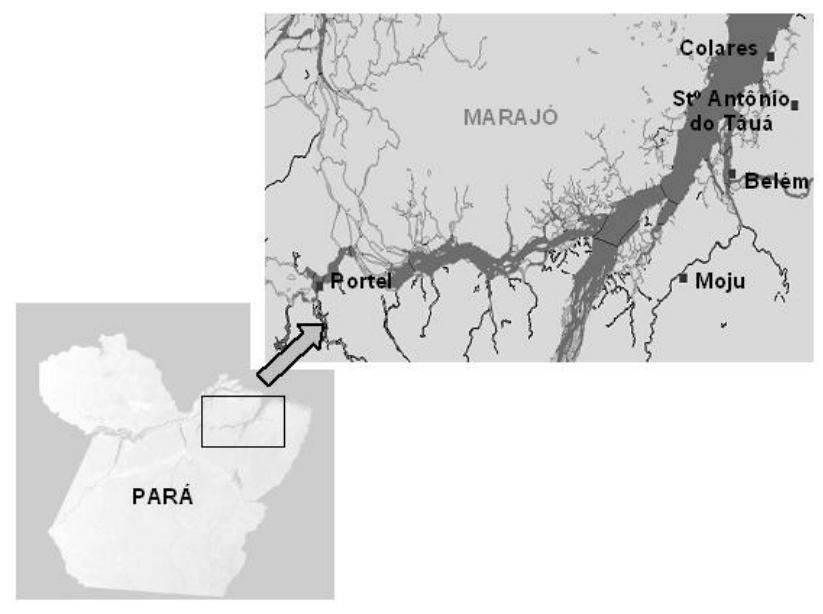

Figura 1 - Localização geográfica das áreas de coleta de frutos bacabi ( 0 . mapora Karsten) e bacaba (0. distichus Mart.) no Estado do Pará.

A caracterização geográfica e climática dos locais de coleta está apresentada na Tabela 1. Foram compilados dados para altitude, coordenadas geográficas (latitude e longitude) e parâmetros climatológicos como: precipitação pluviométrica, temperatura do ar, umidade relativa do ar e insolação anuais e classificados no sistema Köppen, para cada município sede (SUDAM, 1984).

$\mathrm{O}$ experimento foi instalado nos meses de janeiro a maio de 2004 no Instituto de Ciências Agrárias - ICA do campus da Universidade Federal Rural da Amazônia - UFRA, em Belém-PA. Envolveu 47 progênies obtidas a partir da coleta de frutos maduros de matrizes de polinização aberta de plantas que apresentavam tamanho de cacho suficiente para constituir uma amostragem e identificados com uma numeração: 01, 02, $03, \ldots, 47$ para suas respectivas progênies. A espécie $O$. mapora envolveu 38 progênies, sendo 22 procedentes do município de St ${ }^{\circ}$ Antônio do Tauá, 12 de Belém e 4 de Colares, enquanto que a espécie $O$. distichus envolveu 9 progênies, sendo 5 procedentes do município de Portel e 4 de Moju.

Os frutos coletados, foram despolpados manualmente após a imersão por dois dias em água. Somente os frutos que fizeram parte da testemunha (amostra aleatória de sementes de bacabi) 
Tabela 1 - Caracterização geográfica e climática dos locais de coleta de frutos de bacabi (Oenocarpus mapora Karsten) e bacaba (Oenocarpus distichus Mart.) no Estado do Pará.

\begin{tabular}{|c|c|c|c|c|c|c|c|c|}
\hline \multirow{2}{*}{ Municípios } & \multicolumn{8}{|c|}{ Caracterização } \\
\hline & LAT.(S) & LONG.(W) & $\operatorname{ALT} .(m)$ & $\mathrm{PP}(\mathrm{mm})$ & TEMP. $\left({ }^{\circ} \mathrm{C}\right)$ & UR (\%) & INS.(h) & Köppen \\
\hline Belém & $1^{\circ} 22^{\prime} 30^{\prime \prime}$ & $48^{\circ} 37^{\prime} 30^{\prime \prime}$ & 16 & 3.000 & 25 & 85 & 2.200 & Af1 \\
\hline Colares & $0^{\circ} 55^{\prime} 38^{\prime \prime}$ & $48^{\circ} 17^{\prime} 04^{\prime \prime}$ & 3 & 2.500 & $25-26$ & 85 & 2.200 & Am2 \\
\hline Moju & $1^{0} 53^{\prime} 10^{\prime \prime}$ & $48^{\circ} 46^{\prime} 00^{\prime \prime}$ & 18 & 2.250 & 26 & 85 & 2.200 & Af1 \\
\hline Portel & $1^{0} 55^{\prime} 45^{\prime \prime}$ & $50^{\circ} 49^{\prime} 15^{\prime \prime}$ & 4 & 2.200 & $25-26$ & 85 & 2.200 & Af3 \\
\hline St ${ }^{0}$ Ant. do Tauá & $1^{0} 09^{\prime} 06^{\prime \prime}$ & $48^{\circ} 08^{\prime} 00^{\prime}$ & 8 & 2.350 & $25-26$ & 85 & 2.200 & Af2 \\
\hline
\end{tabular}

LAT. : latitude (sul); LONG. : Iongitude (oeste); ALT. : altitude; PP: precipitação pluviométrica; TEMP. : temperatura do ar; UR: umidade relativa do ar; INS. : insolação.

passaram por um tratamento pré-germinativo para quebra de dormência (choque térmico por imersão em água $\pm 50{ }^{\circ} \mathrm{C}$ por 2 horas) (Brasil, 1992). Em seguida as sementes foram semeadas em tubetes acomodados em grades suspensas por bancada a uma altura de $70 \mathrm{~cm}$ do solo no interior do viveiro coberto com sombrite a 50\% de interceptação de luz.

O delineamento experimental foi em blocos ao acaso com quatro repetições de 24 sementes por progênie, sendo as sementes dispostas horizontalmente e a $2 \mathrm{~cm}$ de profundidade. O substrato dos tubetes era constituído por uma mistura de terriço, esterco de ave e resíduo de dendê em proporçóes volumétricas iguais, com irrigação efetuada diariamente.

No estádio de emergência foram tomados diariamente dados percentuais para todas as parcelas em cada progênie e também os dados referentes ao número de plântulas emergidas a cada dia pelo número de dias após a semeadura, conforme as Regras de Análise de Sementes (Brasil, 1992).

A mensuração da base do coleto e altura das plântulas ocorreu ao fim do período emergencial usando como critério a emissão dos folíolos no ápice do caulículo em forma de "palito" em torno de 30 a 40 dias após a emergência. Passados 60 dias após o fim do período emergencial foi realizada nova mensuração do diâmetro e altura das plântulas.

Os dados de percentagem de emergência (PE) foram transformados para arco-seno, segundo a expressão proposta por Snedecor (1945); o tempo médio de emergência (TME) das plântulas foi estimado segundo a equação de Edmond e Drapala (1958) e a Taxa de crescimento absoluto para altura e diâmetro de plântulas (TCAA e TCAD) conforme o descrito por Benincasa (1988).

As análises de variância foram realizadas segundo os procedimentos comuns a um delineamento experimental de blocos casualizados, utilizando o programa GENES desenvolvido por Cruz (2001) o qual emprega os estimadores dos parâmetros genéticos, fenotípicos e os ganhos de seleção esperados, apresentados por Vencovsky \& Barriga (1992). Para as análises de variância tomaram-se dados médios entre parcelas para todas as características e os dados médios com informaçóes dentro das parcelas somente para os caracteres de crescimento. Neste caso, o valor $Y_{\mathrm{ijk}}$, referente à observação na k-ésima planta, da i-ésima progênie e do j-ésimo bloco, é dado por Cruz; Regazzi \& Carneiro (2004).

$$
Y_{i j k}=\mu+G_{i}+B_{j}+\varepsilon_{i j}+\delta_{i j k}
$$

Em que: $\mu=$ média geral; $G_{i}=$ efeito do i-ésimo progênie $(\mathrm{i}=1,2, \ldots, \mathrm{g}, \mathrm{com} \mathrm{g}=47$, decomposto em g' = 38 e g" = 9, sendo $\mathrm{G}_{\mathrm{i}} \sim \operatorname{NID}\left(0, \sigma_{\mathrm{g}}^{2}\right),\left(0, \sigma_{\mathrm{g}}^{2}\right)$ e $\left.\left(0, \sigma_{\mathrm{g}}^{2}\right)\right) ; \mathrm{B}_{\mathrm{j}}=$ efeito do j-ésimo bloco, $\left(j=1,2, \ldots, r, \operatorname{com} r=4\right.$, sendo $B_{j} \sim \operatorname{NID}(0$, $\left.\left.\sigma^{2}{ }_{\mathrm{b}}\right)\right) ; \varepsilon_{\mathrm{ij}}=$ efeito aleatório ambiental existente entre parcelas, sendo $\left.\varepsilon_{\mathrm{ij}} \sim \operatorname{NID}\left(0, \sigma_{\mathrm{e}}^{2}\right)\right) ; \delta_{\mathrm{ijk}}=$ efeito aleatório existente entre plantas dentro das parcelas $\left(\mathrm{k}=1,2, \ldots, \operatorname{com} \mathrm{k}=5\right.$, sendo $\delta_{\mathrm{ijk}}$ - NID $\left(0, \sigma_{d}^{2}\right)$ ). Para o modelo considerado, o esquema da análise de variância é apresentado na Tabela 2 .

Tabela 2 - Esquema da análise de variância de experimento em blocos ao acaso com informação de plantas entre e dentro das parcelas, com as respectivas esperanças dos quadrados médios.

\begin{tabular}{|c|c|c|c|c|}
\hline \multirow{2}{*}{ FV } & \multirow{2}{*}{ G.L } & \multirow{2}{*}{ QM } & \multicolumn{2}{|c|}{$\mathrm{E}(\mathrm{QM})$} \\
\hline & & & Média & Individual \\
\hline Blocos & $r-1$ & QMB & $\cdots$ & $\cdots$ \\
\hline Progênies $(P)$ & $g-1$ & QMP & $\sigma^{2}{ }_{e}+r \sigma_{g}^{2}$ & $\sigma_{d}^{2}+n \sigma_{e}^{2}+n r \sigma_{g}^{2}$ \\
\hline Bacabi $\left(P_{1}\right)$ & $g^{\prime}-1$ & $\mathrm{QMP}_{1}$ & $\sigma^{2}{ }_{e}+r \sigma_{g^{\prime}}^{2}$ & $\sigma_{d}^{2}+n \sigma_{e}^{2}+n r \sigma_{g}^{2}$ \\
\hline Bacaba $\left(P_{2}\right)$ & $g^{\prime \prime}-1$ & $\mathrm{QMP}_{2}$ & $\sigma^{2}{ }_{e}+r \sigma_{g \prime}^{2}$ & $\sigma^{2}{ }_{d}+n \sigma_{e}^{2}+n r \sigma_{g "}^{2}$ \\
\hline$P_{1} v s P_{2}$ & 1 & $\mathrm{QMP}_{1}$ vs $\mathrm{P}_{2}$ & $\ldots$ & $\ldots$ \\
\hline Erro entre & $(r-1)(g-1)$ & QMR & $\sigma^{2}{ }_{e}$ & $\sigma_{d}^{2}+n \sigma_{e}^{2}$ \\
\hline Erro dentro & $(n-1) g r$ & QMD & $\ldots$ & $\sigma_{d}^{2}$ \\
\hline
\end{tabular}




\section{RESULTADOS E DISCUSSÃO}

Foram encontradas diferenças altamente significativas ao nível de $1 \%$ pelo teste $\mathrm{F}$ para as 47 progênies (total), para as 38 progênies de bacabi $(O$. mapora) e para as 9 de bacaba $(O$. distichus) em todos os caracteres avaliados, havendo indicativo da presença de variabilidade significativa entre as progênies, com possibilidade de auxiliar na identificação de indivíduos desejáveis para os caracteres de emergência e crescimento inicial de plântulas (Tabela 3). essa característica, 14 progênies tiveram as melhores taxas, com médias que não diferiram entre si pelo critério ou teste de Scott-Knott $(p>0,05)$. A testemunha (com tratamento para quebra de dormência) foi enquadrada no grupo de melhor desempenho, apresentando média de 94,5\%. Os dados indicam existência de dormência para a maioria das sementes de bacaba e bacabi mas, apesar disto, é possível selecionar progênies que não apresentem dormência. Foram encontradas 12 progênies com média igual ou superior à

Tabela 3 - Quadrados médios para os caracteres de percentagem de emergência (PE), tempo médio de emergência (TME) e taxa de crescimento absoluto da altura e diâmetro de plântulas (TCAA e TCAD) em progênies de bacabi (Oenocarpus mapora Karsten) e bacaba (Oenocarpus distichus Mart.). Belém, Pará, 2004

\begin{tabular}{|c|c|c|c|c|c|}
\hline \multirow{2}{*}{ FV } & \multirow{2}{*}{ G.L } & \multicolumn{4}{|c|}{ Quadrados Médios } \\
\hline & & $\mathrm{PE}^{1}(\%)$ & TME (dias) & TCAA (cm/dia) & TCAD (mm/dia) \\
\hline Progênies (P) & 46 & $793,6312^{* *}$ & $30,950851^{\star *}$ & $0,010831^{\star *}$ & $0,000382^{\star *}$ \\
\hline Bacabi $\left(P_{1}\right)$ & 37 & $886,374976^{* *}$ & $35,79531^{\star \star}$ & $0,009679^{* *}$ & $0,000294^{\star *}$ \\
\hline Bacaba $\left(P_{2}\right)$ & 8 & $443,197939^{* *}$ & $9,341783^{\star *}$ & $0,003572^{* *}$ & $0,000341^{* *}$ \\
\hline$P_{1}$ vs $P_{2}$ & 1 & 165,57756 & $24,578401^{* *}$ & $0,111555^{* *}$ & $0,004005^{* *}$ \\
\hline Entre parcelas & 138 & 64,88764 & 5,061636 & 0,000761 & 0,000119 \\
\hline Dentro de parcelas & 752 & $\ldots$ & $\ldots$ & 0,000474 & 0,00008 \\
\hline Média Geral & $\ldots$ & 62,663 & 30,762 & 0,077 & 0,014 \\
\hline Média $\left(\mathrm{P}_{1}\right)$ & $\ldots$ & 63,120 & 30,586 & 0,083 & 0,015 \\
\hline Média $\left(\mathrm{P}_{2}\right)$ & $\ldots$ & 60,735 & 31,505 & 0,055 & 0,010 \\
\hline
\end{tabular}

**, *: Significativo ao nível de 1\% e 5\% de probabilidade pelo teste F; 1/ PE: Percentagem de emergência transformado para arc sen $\sqrt{ } \% / 100$.

Mantovani (1989) argumenta que a germinação e o estabelecimento de plântulas se caracterizam como as fases mais delicadas do ciclo de vida de uma espécie vegetal, na qual ocorrem as mais altas taxas de mortalidade. Sendo assim, é de se esperar que esta fase sofra intensa pressão de seleção, o que justifica a presença de variação para germinação.

$\mathrm{Na}$ Tabela 4, os valores médios para os quatro caracteres apresentaram a formação de números distintos de grupos de acordo com o critério ou teste de Scott-Knott, indicando a capacidade diferenciadora de alguns caracteres em discriminar divergências genéticas entre os genótipos. Em ordem decrescente de formação de grupos, temos: cinco grupos (A até E) para o caráter tempo médio de emergência (TME); quatro grupos (A até D) para percentagem de emergência (PE); dois grupos (A e B) para a taxa de crescimento absoluto da altura de plântulas (TCAA); e apenas um grupo (A) para o caráter taxa de crescimento absoluto do diâmetro de plântulas (TCAD). Portanto, TME e PE foram os melhores caracteres para representar a variação genética entre as progênies.

$\mathrm{Na}$ fase de emergência houve variabilidade entre as progênies para o caráter $\mathrm{PE}$, verificando intervalo de variação de $27,9 \%$ a $98,9 \%$ e média geral de $75,8 \%$. Para testemunha sendo que, entre estas, onze eram de bacabi e apenas uma de bacaba.

Quanto ao caráter TME, este apresentou intervalo de variação de 24,4 a 37,8 dias entre as progênies, com média geral de 30,76 dias. A testemunha conferiu valor médio de 28,1 dias, mostrando que não se enquadra no grupo com melhor desempenho e indicando que para o caráter TME também é possível selecionar progênies com tempo de emergência mais rápido, evitando o trabalho de quebra de dormência. Esses resultados são coerentes com os relatados por Mariot et al. (1997); Martins-Corder \& Quadros (2001); Zambiazi \& Martins-Corder (2001) para Euterpe edulis. Essas altas percentagens de emergência (germinação e vigor) podem estar relacionadas ao uso de sementes recém-colhidas (Bovi et al., 1988). Esta afirmativa parece óbvia considerando que as sementes são recalcitrantes, ou seja, não podem ser armazenadas por um tempo maior visto que estarão sujeitos ao dessecamento e a perda da viabilidade, portanto pode ser dispensável.

Com relação ao crescimento, o caráter TCAA apresentou intervalo de variação de 0,037 a $0,134 \mathrm{~cm} /$ dia com média geral de $0,077 \mathrm{~cm} /$ dia. Foi verificado no grupo A similaridade entre 
Tabela 4 - Comparação de médias para os caracteres de percentagem de emergência (PE), tempo médio de emergência (TME) e taxa de crescimento absoluto da altura e diâmetro de plântulas (TCAA e TCAD) em progênies de bacabi (Oenocarpus mapora Karsten) e bacaba (Oenocarpus distichus Mart.). Belém, Pará, 2004

\begin{tabular}{|c|c|c|c|c|c|c|c|c|}
\hline \multicolumn{9}{|c|}{ CARACTERES } \\
\hline \multicolumn{3}{|c|}{ PE1 } & \multicolumn{2}{|c|}{ TME (dias) } & \multicolumn{2}{|c|}{ TCAA (cm/dia) } & \multicolumn{2}{|c|}{ TCAD (mm/dia) } \\
\hline Progênie & $\%$ & Média & Progênie & Média & Progênie & Média & Progênie & Média \\
\hline 29 bacabi & 98,9 & $84,08 \mathrm{a}$ & 03 bacabi & $24,45 a$ & 11 bacabi & $0,134 \mathrm{a}$ & 09 bacabi & $0,022 a$ \\
\hline 12 bacabi & 98,9 & $84,08 \mathrm{a}$ & 09 bacabi & $25,58 \mathrm{a}$ & 13 bacabi & $0,121 \mathrm{a}$ & 08 bacabi & $0,021 \mathrm{a}$ \\
\hline 10 bacabi & 98,4 & $82,85 \mathrm{a}$ & 02 bacabi & $25,92 \mathrm{a}$ & 14 bacabi & $0,118 \mathrm{a}$ & 16 bacabi & $0,021 \mathrm{a}$ \\
\hline 03 bacabi & 98,4 & $82,85 \mathrm{a}$ & 10 bacabi & $27,27 \mathrm{~b}$ & 09 bacabi & $0,116 \mathrm{a}$ & 15 bacabi & $0,020 a$ \\
\hline 27 bacaba & 97,8 & $81,62 \mathrm{a}$ & 35 bacabi & $27,84 \mathrm{~b}$ & 17 bacabi & $0,112 \mathrm{a}$ & 03 bacabi & $0,020 \mathrm{a}$ \\
\hline 02 bacabi & 95,8 & $78,17 \mathrm{a}$ & 47 bacabi & $27,93 \mathrm{~b}$ & 12 bacabi & $0,111 \mathrm{a}$ & 37 bacabi & $0,020 \mathrm{a}$ \\
\hline 39 bacabi & 95,7 & $78,05 \mathrm{a}$ & 34 bacabi & $28,11 b$ & 16 bacabi & $0,104 \mathrm{a}$ & 11 bacabi & $0,019 a$ \\
\hline 09 bacabi & 95,7 & $78,05 \mathrm{a}$ & 08 bacabi & $28,41 \mathrm{~b}$ & 07 bacabi & $0,101 \mathrm{a}$ & 35 bacabi & $0,019 a$ \\
\hline 01 bacabi & 95,6 & $77,97 \mathrm{a}$ & 14 bacabi & $28,44 \mathrm{~b}$ & 43 bacabi & $0,097 \mathrm{a}$ & 12 bacabi & $0,018 a$ \\
\hline 28 bacabi & 94,8 & $76,94 \mathrm{a}$ & 38 bacabi & $28,53 \mathrm{~b}$ & 44 bacabi & $0,095 \mathrm{a}$ & 01 bacabi & $0,018 \mathrm{a}$ \\
\hline 43 bacabi & 94,8 & $76,82 \mathrm{a}$ & 32 bacabi & $28,67 \mathrm{~b}$ & 06 bacabi & $0,093 \mathrm{a}$ & 46 bacabi & $0,017 \mathrm{a}$ \\
\hline 47 bacabi & 94,5 & $76,45 \mathrm{a}$ & 24 bacaba & $28,71 \mathrm{~b}$ & 33 bacabi & $0,089 a$ & 39 bacabi & $0,017 \mathrm{a}$ \\
\hline 04 bacabi & 92,8 & $74,47 \mathrm{a}$ & 45 bacabi & $29,16 \mathrm{c}$ & 08 bacabi & $0,087 \mathrm{a}$ & 04 bacabi & $0,017 \mathrm{a}$ \\
\hline 16 bacabi & 91,5 & $73,11 \mathrm{a}$ & 11 bacabi & $29,18 \mathrm{c}$ & 04 bacabi & $0,085 \mathrm{a}$ & 22 bacaba & $0,017 \mathrm{a}$ \\
\hline 21 bacaba & 89,3 & $70,97 \mathrm{~b}$ & 28 bacabi & $29,36 \mathrm{c}$ & 35 bacabi & $0,085 a$ & 28 bacabi & $0,017 \mathrm{a}$ \\
\hline 13 bacabi & 88,1 & $69,90 \mathrm{~b}$ & 40 bacabi & $29,44 \mathrm{C}$ & 18 bacabi & $0,085 \mathrm{a}$ & 13 bacabi & $0,017 \mathrm{a}$ \\
\hline 11 bacabi & 87,9 & 69,66 b & 05 bacabi & $29,45 \mathrm{C}$ & 10 bacabi & $0,085 a$ & 33 bacabi & $0,017 \mathrm{a}$ \\
\hline 17 bacabi & 87,3 & $69,19 \mathrm{~b}$ & 36 bacabi & $29,56 \mathrm{c}$ & 37 bacabi & $0,084 \mathrm{a}$ & 17 bacabi & $0,016 \mathrm{a}$ \\
\hline 46 bacabi & 84,6 & $66,97 \mathrm{~b}$ & 43 bacabi & $29,66 \mathrm{c}$ & 47 bacabi & $0,083 \mathrm{a}$ & 36 bacabi & $0,016 a$ \\
\hline 23 bacaba & 84,0 & $66,46 \mathrm{~b}$ & 07 bacabi & $29,92 \mathrm{c}$ & 36 bacabi & $0,083 \mathrm{a}$ & 07 bacabi & $0,016 \mathrm{a}$ \\
\hline 45 bacabi & 83,9 & $66,35 \mathrm{~b}$ & 46 bacabi & $30,02 \mathrm{c}$ & 03 bacabi & $0,082 \mathrm{a}$ & 18 bacabi & $0,016 a$ \\
\hline 15 bacabi & 82,4 & $65,22 \mathrm{~b}$ & 15 bacabi & $30,07 \mathrm{c}$ & 38 bacabi & $0,080 \mathrm{a}$ & 43 bacabi & $0,015 a$ \\
\hline 44 bacabi & 81,7 & $64,68 \mathrm{~b}$ & 19 bacaba & 30,26 c & 45 bacabi & $0,079 \mathrm{a}$ & 10 bacabi & $0,015 a$ \\
\hline 07 bacabi & 80,3 & $63,69 \mathrm{~b}$ & 39 bacabi & $30,57 \mathrm{c}$ & 28 bacabi & $0,077 \mathrm{~b}$ & 34 bacabi & $0,015 a$ \\
\hline 31 bacabi & 79,2 & $62,90 \mathrm{~b}$ & 01 bacabi & $30,73 \mathrm{c}$ & 01 bacabi & $0,076 \mathrm{~b}$ & 45 bacabi & $0,014 \mathrm{a}$ \\
\hline 30 bacabi & 78,3 & $62,26 \mathrm{~b}$ & 30 bacabi & $30,92 \mathrm{c}$ & 46 bacabi & $0,075 \mathrm{~b}$ & 21 bacaba & $0,014 \mathrm{a}$ \\
\hline 22 bacaba & 77,2 & $61,51 \mathrm{~b}$ & 21 bacaba & $30,92 \mathrm{c}$ & 32 bacabi & $0,074 \mathrm{~b}$ & 40 bacabi & $0,013 a$ \\
\hline 08 bacabi & 75,3 & $60,24 \mathrm{c}$ & 27 bacaba & $30,98 \mathrm{c}$ & 24 bacaba & $0,073 \mathrm{~b}$ & 47 bacabi & $0,013 a$ \\
\hline 32 bacabi & 75,2 & $60,16 \mathrm{c}$ & 31 bacabi & $31,18 \mathrm{c}$ & 30 bacabi & $0,072 \mathrm{~b}$ & 31 bacabi & $0,013 a$ \\
\hline 37 bacabi & 69,9 & $56,74 \mathrm{c}$ & 17 bacabi & $31,22 \mathrm{c}$ & 39 bacabi & $0,072 \mathrm{~b}$ & 24 bacaba & $0,013 a$ \\
\hline 25 bacaba & 69,7 & $56,62 \mathrm{c}$ & 23 bacaba & $31,57 d$ & 25 bacaba & $0,070 \mathrm{~b}$ & 44 bacabi & $0,013 a$ \\
\hline 24 bacaba & 66,5 & $54,68 \mathrm{c}$ & 22 bacaba & $31,97 \mathrm{~d}$ & 22 bacaba & $0,066 \mathrm{~b}$ & 02 bacabi & $0,013 a$ \\
\hline 14 bacabi & 65,9 & $54,31 \mathrm{c}$ & 25 bacaba & $32,02 \mathrm{~d}$ & 40 bacabi & $0,064 \mathrm{~b}$ & 32 bacabi & $0,012 \mathrm{a}$ \\
\hline 19 bacaba & 64,8 & $53,65 \mathrm{c}$ & 16 bacabi & $32,16 \mathrm{~d}$ & 21 bacaba & $0,064 \mathrm{~b}$ & 38 bacabi & $0,012 \mathrm{a}$ \\
\hline 35 bacabi & 64,6 & $53,51 \mathrm{c}$ & 29 bacabi & $32,82 \mathrm{~d}$ & 15 bacabi & $0,060 \mathrm{~b}$ & 23 bacaba & $0,012 a$ \\
\hline 18 bacabi & 61,5 & $51,66 \mathrm{c}$ & 42 bacabi & $32,83 d$ & 34 bacabi & $0,059 \mathrm{~b}$ & 29 bacabi & $0,012 \mathrm{a}$ \\
\hline 26 bacaba & 59,6 & $50,55 \mathrm{c}$ & 04 bacabi & $33,25 d$ & 02 bacabi & $0,058 \mathrm{~b}$ & 30 bacabi & $0,012 a$ \\
\hline 20 bacaba & 59,5 & $50,51 \mathrm{c}$ & 33 bacabi & $33,30 \mathrm{~d}$ & 42 bacabi & $0,056 \mathrm{~b}$ & 14 bacabi & $0,011 \mathrm{a}$ \\
\hline 40 bacabi & 58,4 & $49,86 \mathrm{c}$ & 20 bacaba & $33,40 \mathrm{~d}$ & 31 bacabi & $0,056 \mathrm{~b}$ & 06 bacabi & $0,010 \mathrm{a}$ \\
\hline 36 bacabi & 54,2 & $47,43 \mathrm{c}$ & 44 bacabi & $33,51 \mathrm{~d}$ & 05 bacabi & $0,054 \mathrm{~b}$ & 25 bacaba & $0,010 \mathrm{a}$ \\
\hline 42 bacabi & 51,0 & $45,58 d$ & 26 bacaba & $33,66 \mathrm{~d}$ & 23 bacaba & $0,053 \mathrm{~b}$ & 42 bacabi & $0,010 \mathrm{a}$ \\
\hline 38 bacabi & 50,0 & $45,00 d$ & 37 bacabi & $33,76 \mathrm{~d}$ & 20 bacaba & $0,044 \mathrm{~b}$ & 05 bacabi & $0,008 \mathrm{a}$ \\
\hline
\end{tabular}


Tabela 4 - Continuação

\begin{tabular}{|c|c|c|c|c|c|c|c|c|}
\hline \multicolumn{9}{|c|}{ CARACTERES } \\
\hline \multicolumn{3}{|c|}{ PE1 } & \multicolumn{2}{|c|}{ TME (dias) } & \multicolumn{2}{|c|}{ TCAA (cm/dia) } & \multicolumn{2}{|c|}{ TCAD (mm/dia) } \\
\hline 33 bacabi & 48,9 & $44,38 d$ & 18 bacabi & $34,73 \mathrm{e}$ & 19 bacaba & $0,044 \mathrm{~b}$ & 27 bacaba & $0,008 \mathrm{a}$ \\
\hline 05 bacabi & 40,1 & $39,32 d$ & 12 bacabi & 35,36 e & 29 bacabi & $0,043 b$ & 26 bacaba & $0,007 \mathrm{a}$ \\
\hline 34 bacabi & 39,3 & $38,85 d$ & 13 bacabi & 35,36 e & 27 bacaba & $0,042 \mathrm{~b}$ & 19 bacaba & $0,006 \mathrm{a}$ \\
\hline 41 bacabi & 32,4 & $34,72 d$ & 41 bacabi & 35,62 e & 26 bacaba & $0,039 \mathrm{~b}$ & 41 bacabi & $0,006 \mathrm{a}$ \\
\hline 06 bacabi & 27,9 & $31,90 \mathrm{~d}$ & 06 bacabi & $37,80 \mathrm{e}$ & 41 bacabi & $0,037 \mathrm{~b}$ & 20 bacaba & $0,005 \mathrm{a}$ \\
\hline Testemunha & 94,5 & 76,45 & Testemunha & 28,14 & Testemunha & 0,184 & Testemunha & 0,048 \\
\hline Média Geral & 75,8 & 62,66 & Média Geral & 30,76 & Média Geral & 0,077 & Média Geral & 0,014 \\
\hline
\end{tabular}

Médias seguidas da mesma letra não diferem significativamente pelo teste Scott-Knott ao nível de $5 \%$ de probabilidade; 1/ PE: Percentagem de emergência transformado para arc $\operatorname{sen} \sqrt{ } \% / 100$.

as médias em 23 progênies, que não diferem significativamente pelo teste Scott-Knott $(\mathrm{p}>0,05)$. A média das testemunhas, de $0,184 \mathrm{~cm} / \mathrm{dia}$, foi superior a todas as médias das progênies, indicando que a quebra de dormência pode ter favorecido o caráter TCAA.

Para o caráter TCAD, o intervalo de variação oscilou entre 0,005 a $0,022 \mathrm{~mm} /$ dia com média de $0,014 \mathrm{~mm} /$ dia; estas médias não contribuíram para a formação de novos grupos, indicando não haver diferença significativa entre as 47 progênies para este caráter. Quanto à testemunha, esta
A magnitude dos ganhos por seleção e sua facilidade podem ser preditas pelas estimativas dos parâmetros genéticos apresentados na Tabela 5.

$\mathrm{Na}$ obtenção dessas estimativas foi considerada a relação de meio-irmãos nas progênies envolvidas e considerada desprezível a ocorrência de endogamia na população-base em estudo (condição imprescindível preconizada por Vencovsky, 1978).

Para a herdabilidade no sentido amplo foi verificado que no conjunto total de progênies e no conjunto de progênies

Tabela 5 - Parâmetros genéticos e ambientais para os caracteres de percentagem de emergência (PE), tempo médio de emergência (TME) e taxa de crescimento absoluto da altura e diâmetro de plântulas (TCAA e TCAD) em progênies de bacabi (Oenocarpus mapora Karsten) e bacaba (Oenocarpus distichus Mart.). Belém, Pará, 2004.

\begin{tabular}{|c|c|c|c|c|c|c|}
\hline \multirow{2}{*}{ Progênies } & \multirow{2}{*}{ Caracteres } & \multicolumn{5}{|c|}{ Parâmetros } \\
\hline & & $\sigma_{g}^{2}$ & $\mathrm{CV}_{\mathrm{g}}(\%)$ & $\mathrm{CV}_{\mathrm{e}}(\%)$ & $h_{m}^{2}(\%)$ & $\mathrm{CV}_{\mathrm{g}} / \mathrm{CV}_{\mathrm{e}}$ \\
\hline \multirow{4}{*}{ Total (P) } & $\mathrm{PE}^{1}(\%)$ & 182,18589 & 21,54 & 12,85 & 91,82 & 1,67 \\
\hline & TME (dias) & 6,472304 & 8,27 & 7,31 & 83,65 & 1,13 \\
\hline & TCAA (cm/dia) & 0,0005035 & 29,14 & 16,02 & 92,98 & 1,81 \\
\hline & TCAD (mm/dia) & 0,0000135 & 25,90 & 34,84 & 68,84 & 0,74 \\
\hline \multirow{4}{*}{ Bacabi $\left(P_{1}\right)$} & $\mathrm{PE}^{1}(\%)$ & 205,371834 & 22,70 & 12,76 & 92,68 & 1,77 \\
\hline & TME (dias) & 7,683419 & 9,06 & 7,35 & 85,86 & 1,23 \\
\hline & TCAA (cm/dia) & 0,0004459 & 25,44 & 15,32 & 92,14 & 1,66 \\
\hline & TCAD (mm/dia) & 0,0000087 & 19,72 & 32,41 & 59,52 & 0,60 \\
\hline \multirow{4}{*}{ Bacaba $\left(P_{2}\right)$} & $\mathrm{PE}^{1}(\%)$ & 94,577574 & 16,01 & 13,26 & 85,36 & 1,20 \\
\hline & TME (dias) & 1,070036 & 3,28 & 7,14 & 45,82 & 0,45 \\
\hline & TCAA (cm/dia) & 0,0001405 & 21,55 & 17,78 & 78,69 & 1,21 \\
\hline & TCAD (mm/dia) & 0,0000111 & 33,31 & 34,98 & 15,74 & 0,95 \\
\hline
\end{tabular}

1/ PE: Percentagem de emergência transformado para arc sen $\sqrt{ } \% / 100 ; \sigma^{2} \mathrm{~g}$ : variância genética entre progênies; CVg (\%): coeficiente de variação genética entre progênies; CVe (\%): coeficiente de variação ambiental entre progênies; $h^{2} \mathrm{~m}$ : coeficiente de herdabilidade médio; CVg/CVe: índice "b".

alcançou média de $0,048 \mathrm{~mm} /$ dia, mostrando-se superior às outras progênies, o que pode ser explicado pela quebra de dormência que também pode ter favorecido o alto valor para o caráter TCAD. No palmiteiro Martins-Corder \& Quadros (2001) obtiveram resultados semelhantes aos encontrado neste estudo. de bacabi todos os caracteres estudados apresentaram estimativas acima de 50\%. As exceçôes foram para TME e TCAD no conjunto de progênies de bacaba, com estimativas de herdabilidade abaixo de $50 \%$, o que pode ser atribuído ao pequeno tamanho da amostra deste conjunto. Quanto à relação entre os coeficientes de variação genética e ambiental conhecido como índice "b", os valores apresentaram a 
mesma tendência da herdabilidade e revelam alta proporção de variação genética, ou seja, boa condição para selecionar genótipos superiores para estes caracteres, conforme sugeriu Vencovsky (1978).

A Tabela 6 apresenta as estimativas de ganho genético e as médias após um ciclo de seleção quando se usam sementes remanescentes para recombinação. Adotando este procedimento de seleção foi possível obter ganhos de seleção para todos os caracteres estudados, sendo menos expressivos os para TME.

\section{CONCLUSÕES}

Há variação genética significativa entre progênies de bacabi e bacaba para os quatro caracteres, indicando potencial genético a ser explorado de maneira efetiva em programas de melhoramento.

Os caracteres TME e PE foram os que melhor discriminaram, havendo a formação de maior número de grupos comparativamente aos demais caracteres sendo,

Tabela 6 - Ganho genético e média da população após o ciclo de seleção para os caracteres de emergência (PE), tempo médio de emergência (TME) e taxa de crescimento absoluto da altura e diâmetro de plântulas (TCAA e TCAD) em progênies de bacabi (Oenocarpus mapora Karsten) e bacaba (Oenocarpus distichus Mart.). Belém, Pará, 2004.

\begin{tabular}{|c|c|c|c|c|c|}
\hline \multirow{2}{*}{ Progênies } & \multirow{2}{*}{ Caracteres } & \multicolumn{2}{|c|}{ Ganho genético } & \multicolumn{2}{|c|}{ Médias } \\
\hline & & $\mathrm{GS}_{\mathrm{e}}$ & $\%$ & $\mu_{0}$ & $\mu_{\mathrm{s}}$ \\
\hline \multirow{4}{*}{ Total (P) } & $\mathrm{PE}^{1}(\%)$ & 21,7292 & 34,67 & 62,663 & 84,392 \\
\hline & TME (dias) & $-3,9089$ & 12,70 & 30,762 & 26,853 \\
\hline & TCAA (cm/dia) & 0,0363 & 47,14 & 0,077 & 0,113 \\
\hline & TCAD (mm/dia) & 0,0051 & 36,42 & 0,014 & 0,019 \\
\hline \multirow{4}{*}{ Bacabi $\left(P_{1}\right)$} & $\mathrm{PE}^{1}(\%)$ & 21,660 & 34,31 & 63,120 & 84,780 \\
\hline & TME (dias) & $-4,0324$ & 13,18 & 30,586 & 26,553 \\
\hline & TCAA (cm/dia) & 0,0318 & 38,31 & 0,083 & 0,114 \\
\hline & TCAD (mm/dia) & 0,0035 & 23,33 & 0,015 & 0,018 \\
\hline \multirow{4}{*}{ Bacaba $\left(P_{2}\right)$} & $\mathrm{PE}^{1}(\%)$ & 10,8718 & 17,90 & 60,735 & 71,606 \\
\hline & TME (dias) & $-0,8472$ & 2,68 & 31,505 & 30,657 \\
\hline & TCAA (cm/dia) & 0,0127 & 23,09 & 0,055 & 0,067 \\
\hline & TCAD (mm/dia) & 0,0032 & 32,00 & 0,010 & 0,013 \\
\hline
\end{tabular}

Entre progênies: seleção de $10 \%$ do total das progênies $(k=1,68), 10 \%$ das progênies bacabi $(k=1,57)$ e $20 \%$ das progênies bacaba ( $k=1,21)$; $\mu 0$ : média da população original; $\mu \mathrm{s}$ : média da população selecionada; $1 / \mathrm{PE}$ : Percentagem de emergência transformado para arc sen $\sqrt{ } \% / 100$.

No conjunto total de progênies e conjunto de progênies de bacabi, mesmo apresentando amostragens diferentes, todos os caracteres avaliados apresentaram estimativas relativamente altas, favorecidas pela pressão de seleção de $10 \%$ que também foi considerada alta (cinco progênies selecionadas). Em progênies do conjunto de bacaba, como a amostragem era pequena, foi utilizada uma pressão de seleção de $20 \%$ (duas progênies selecionadas) para os caracteres, resultando em estimativas menores conforme o esperado pelas informaçôes de variância genética e herdabilidade no sentido amplo.

Para as espécies estudadas é possível, através de seleção, aumentar a média de percentagem de emergência e reduzir o tempo médio de emergência, aspectos importantes do ponto de vista de domesticação das espécies; entretanto, nossos resultados indicam que isto será mais eficiente para o bacabi. No caso do bacaba, há de se considerar o tamanho pequeno da amostra utilizada. Resultados semelhantes foram encontrados para capacidade e vigor de emergência em guaraná, conforme estudo de Conceição, Mota \& Kato, 1999. portanto, caracteres importantes a serem empregados em estudos envolvendo a diversidade genética das duas espécies.

As progênies que apresentaram as médias mais altas para $\mathrm{PE}$ e as mais baixas para TME, podem ser selecionadas para constituir um grupo de progênies que resultará em um maior sincronismo de emergência, visando aumentar a produtividade econômica através de mudas vigorosas e sadias, sem o uso de quebra de dormência das sementes.

O caráter percentagem de emergência apresentou a maior variação genética, podendo ser essa uma estratégia adaptativa importante para a espécie.

É possível obter ganhos de seleção para vigor de sementes e crescimento inicial de plântulas, quando selecionados entre média de progênies de modo que estes caracteres possam ser indicados na seleção de matrizes mais adaptadas aos sistemas de cultivo. 


\section{BIBLIOGRAFIA CITADA}

Almeida, S. S.; Silva, P. J. D. 1992. Cadastro e sistematização das palmeiras úteis da Amazônia. Encontro de Bolsistas do Museu Paraense Emílio Goeldi. Resumos. Belém, maio: 26.

Balick, M. J. 1986. Systematics and economic botany of the Oenocarpus-Jessenia (Palmae) complex. Advances in Economic Botany, 3:1-140.

Balick, M. J. 1992. Jessenia y Oenocarpus: plantas aceiteros neotropicales dignas de ser domesticadas. In: (autores) Estudio FAO produccion y proteccion vegetal 88 . Organizacion de las naciones unidas para la agricultura y la alimentacion. Roma. $180 \mathrm{p}$.

Benincasa, M. M. P. 1988. Análise do crescimento de plantas: (noções básicas). Jaboticabal, FUNEP, 42 p. (Boletim Técnico 467a).

Bovi, M. L. A. et al. 1988. Pesquisas com o gênero Euterpe e Bactris no Instituto Agronômico de Campinas. In: ENCONTRO NACIONAL DE PESQUISADORES EM PALMITO, 1., Curitiba, 1988, Anais..., Curitiba: Embrapa-CNPF, p. 1-43 (Embrapa-CNPF, Documento, 19).

Brasil. 1992. Ministério da Agricultura e Reforma Agrária. Regras para análise de sementes. SNDA/DNDV/CLAV. Brasília, 365 p.

Conceição, C. C. C. da; Mota, M. G. da C.; Kato, A. K. 1999. Estimativas de parâmetros genéticos para germinação de sementes de guaraná (Paullinia cupana var. sorbilis (Mart.) Ducke). Rev. Ciênc. Agrár, Belém, n. 32, p. 47-53, jul./dez.

Cruz, C. D. 2001. Programa Genes: versão Windows, aplicativo computacional em genética e estatística. Viçosa, MG: UFV, 648 pp.

Cruz, C. D.; Regazzi, A. J.; Carneiro, P. C. S. 2004. Modelos biométricos aplicados ao melhoramento genético. 3. ed. Viçosa, MG: UFV, 1 v. 480 pp.

Cunha, A. C. da C.; Jardim, M. A. G. 1995. Avaliação do potencial germinativo em açaí (Euterpe oleracea Mart.). Variedades preto, branco e espada. In: I Reunião dos Botânicos da Amazônia, 26 a 30 jun. Belém: PA, 1995. Boletim do Museu Paraense Emílio Goeldi, sér. Bot. 11(1), p. 55-59.

Edmond, J. B.; Drapala, W. J. 1958. The efffects of temperature, sand and soil, and acetone on germination of okra seed. Proceedings of the American Society for Horticultural Science, Leuven, v.71, p. 428-434.

Lleras, E; Giacometti, D. C.; Coradin. 1983. Áreas críticas de distribución de palmas en las Americas para colecta, evalución y conservación. In: Reunión de Consulta sobre Palmeras poco Utilizadas de America Tropical, Turrialba. Informe..., Turrialba: FAO/CATIE, p. 67-101.
Mantovani, W. 1989. Dinâmica de populações. Simpósio Mata Ciliar. Anais..., Campinas-SP, p. 120-129.

Mariot, A.; Reis, M. S. dos, Nodari, R. O. 1997. Variação genética em progênies de populações naturais de Euterpe edulis Mart. proveniente de diferentes altitudes. In: CONGRESSO NACIONAL DE GENÉTICA, Caxambu, 1997. Anais..., Caxambu: SBG, p. 326.

Martins-Corder, M. P.; Quadros, V. R. B. 2001. Análise do vigor de sementes e crescimento de plântulas de distintas progênies de Euterpe edulis Mart. In: SIMPÓSIO DE RECURSOS GENÉTICOS PARA AMÉRICA LATINA E CARIBE, v. 3, Londrina, 2001. Anais..., Londrina: IAPAR, p. 537-538.

Nascimento, W. M. O. et al. 2001. Influência da posição de semeadura sobre a germinação, vigor e crescimento de plântulas de bacabinha (Oenocarpus mapora Karsten - Arecaceae). Belém: Embrapa CPATU, v. 11 n. 2 set., p. 328-328 (Informativo ABRATES).

Oliveira, J. et al. 1991. Espécies vegetais produtoras de fibras utilizadas por comunidades amazônicas. Boletim do Museu Paraense Emílio Goeldi, sér. Bot. 7(2): p. 393-428.

Pinheiro, C. U. B. 1986. Germinação de sementes de palmeiras: Revisão bibliográfica. Teresina, $102 \mathrm{pp}$.

Snedecor, G. W. 1945. Métodos estatísticos. Lisboa: Ministério da Economia, 469 pp.

SUPERITENDÊNCIA DO DESENVOLVIMENTO DA AMAZÔNIA. 1984. Atlas climatológico da Amazônia. Belém: SUDAM, Projeto de Hidrologia e climatologia da Amazônia, $125 \mathrm{pp}$.

Vencovsky, R. 1978. Herança quantitativa. In.: Paterniani, E, Viégas, G. P. (Eds.). Melhoramento e produção do milho. 2. ed. Campinas: Fundação Cargill, v. 1, p. 137-214.

Vencovsky, R.; Barriga, P. 1992. Genética biométrica no fitomelhoramento. Revista Brasileira de Genética, Ribeirão Preto: SP. p. 496.

Zambiazi, D. C.; Martins-Corder, M. P. 2001. Variabilidade genética de progênies de palmiteiro (Euterpe edulis Martius ARECACEAE) para vigor germinativo. In: III SIMPÓSIO DE RECURSOS GENÉTICOS PARA A AMÉRICA LATINA E CARIBE, v. 3, Londrina, 2001. Anais..., Londrina: IAPAR, p. 453-455.

Recebido em 27/11/2007

Aceito em 11/05/2009 